\title{
An Astrocyte-Flow Mapping on a Mesh-Based Communication Infrastructure to Defective Neurons Phagocytosis
}

\author{
Amir Masoud Rahmani ${ }^{1,+}{ }^{+}$, Rizwan Ali Naqvi ${ }^{2,+}{ }^{\mathbb{D}}$, Saqib Ali ${ }^{3}$, , Seyedeh Yasaman Hosseini Mirmahaleh ${ }^{4,5} \mathbb{D}$, \\ Mohammed Alswaitti ${ }^{6, *(\mathbb{D})}$, Mehdi Hosseinzadeh ${ }^{7, *(\mathbb{D})}$ and Kamran Siddique ${ }^{6}(\mathbb{D})$ \\ 1 Future Technology Research Center, National Yunlin University of Science and Technology, \\ Douliou 64002, Taiwan; rahmania@yuntech.edu.tw \\ 2 Department of Intelligent Mechatronics Engineering, Sejong University, Seoul 05006, Korea; \\ rizwanali@sejong.ac.kr \\ 3 Department of Information Systems, College of Economics and Political Science, Sultan Qaboos University, \\ Muscat 123, Oman; saqib@squ.edu.om \\ 4 Department of Electrical Engineering, Science and Technology, Lille University, 59000 Lille, France; \\ Yasaman-Hosseini@ieee.org \\ 5 Department of Computer Engineering, Science and Research Branch, Islamic Azad University, \\ Tehran 021, Iran \\ 6 Department of Information and Communication Technology, School of Electrical and Computer Engineering, \\ Xiamen University Malaysia, Sepang 43900, Malaysia; kamran.siddique@xmu.edu.my \\ 7 Pattern Recognition and Machine Learning Lab, Gachon University, 1342 Seongnamdaero, Sujeanggu, \\ Seongnam 13120, Korea \\ check for \\ updates \\ * Correspondence: alswaitti.mohammed@xmu.edu.my (M.A.); mehdi@gachon.ac.kr (M.H.) \\ † Amir Masoud Rahmani and Rizwan Ali Naqvi have contributed equally to this work.
}

Citation: Rahmani, A.M.; Ali Naqvi, R.; Ali, S.; Hosseini Mirmahaleh, S.Y.; Alswaitti, M.; Hosseinzadeh, M.; Siddique, K. An Astrocyte-Flow Mapping on a Mesh-Based

Communication Infrastructure to Defective Neurons Phagocytosis. Mathematics 2021, 9, 3012. https:// doi.org/10.3390/math9233012

Academic Editor:

Alessandro Niccolai

Received: 9 October 2021

Accepted: 18 November 2021

Published: 24 November 2021

Publisher's Note: MDPI stays neutral with regard to jurisdictional claims in published maps and institutional affiliations.

Copyright: (c) 2021 by the authors. Licensee MDPI, Basel, Switzerland. This article is an open access article distributed under the terms and conditions of the Creative Commons Attribution (CC BY) license (https:/ / creativecommons.org/licenses/by/ $4.0 /)$.

\begin{abstract}
In deploying the Internet of Things (IoT) and Internet of Medical Things (IoMT)-based applications and infrastructures, the researchers faced many sensors and their output's values, which have transferred between service requesters and servers. Some case studies addressed the different methods and technologies, including machine learning algorithms, deep learning accelerators, Processing-In-Memory (PIM), and neuromorphic computing (NC) approaches to support the data processing complexity and communication between IoMT nodes. With inspiring human brain structure, some researchers tackled the challenges of rising IoT- and IoMT-based applications and neural structures' simulation. A defective device has destructive effects on the performance and cost of the applications, and their detection is challenging for a communication infrastructure with many devices. We inspired astrocyte cells to map the flow (AFM) of the Internet of Medical Things onto mesh network processing elements (PEs), and detect the defective devices based on a phagocytosis model. This study focuses on an astrocyte's cholesterol distribution into neurons and presents an algorithm that utilizes its pattern to distribute IoMT's dataflow and detect the defective devices. We researched Alzheimer's symptoms to understand astrocyte and phagocytosis functions against the disease and employ the vaccination COVID-19 dataset to define a set of task graphs. The study improves total runtime and energy by approximately $60.85 \%$ and $52.38 \%$ after implementing AFM, compared with before astrocyte-flow mapping, which helps IoMT's infrastructure developers to provide healthcare services to the requesters with minimal cost and high accuracy.
\end{abstract}

Keywords: flow mapping; defective device detection; mesh network; astrocyte cells; phagocytosis

\section{Introduction}

The complexity of the relationship between service providers and requesters and balancing the flow between them is challenging for IoT- and IoMT-based infrastructures. When sharing a patient's private information on the Internet of Medical Things, intruders may be able to access their records, leading to security and confidentiality risks. A defective device also deteriorates the security by distributing malicious messages and data, in 
which the issue endangers the patient's health situation [1,2]. Some studies presented different approaches and protocols to follow doctor authentication, client privacy, and creating security purposes for the various IoMT-based applications [1-3]. The researchers aimed their case studies on dependability and availability and provided different fault detection methods to protect people's confidential information and improve the quality of service. Some studies inspired the human brain's neural cells to present the different approaches for improving the performance and cost of the IoT and IoMT applications in facing the increasing complexity of their computational operations and the relationship between their components and layers [4-6]. Kwon et al. [7-9] addressed many hardwaredeep learning accelerators (DLAs) to tackle the energy efficiency problem in the graphic processing unit (GPU)-based systems and support the complexity of implementing different machine learning algorithms (convolutional neural networks, deep neural networks, and recurrent neural networks) that were employed in the IoMT physical layer, and improved its performance and cost. The researchers utilized the deep neural network (DNN) and reinforcement learning to decision-making about the service providers in the IoT by rewardbased agents, which their studies inspired from the hieratical structure of the human brain [10-12]. Guan et al. [13], utilized the NC's memristor feature to the processing in a resistive random-access memory (ReRAM), in which neuromorphic computing-PIM affects improving energy efficiency and communication delay because of reducing memory accesses. The processing-in-memory affects the improvement of IoMT's efficiency as employing neuromorphic computing in PIM has a highlighted role in order to speedup computational operation and reduce the cost $[14,15]$. Additionally, the researcher simulated the body language (such as hand sign language) through the neuromorphic computing that was inspired by the human neural structures [16].

By reviewing Alzheimer's disease and its internal symptoms, the researchers demonstrated the astrocytes cells' highlighted role in the disease progression that related to distributing the cholesterol into the neuron [17]. The astrocyte cells diagnosis the dead cell and waste by phagocytosis operation and decomposition and digestion them to remove their negative impact. The medical studies proved astrocytes' vital role in the nutrition of neurons that lack fairness distributing the cholesterols and creating amyloid plaques have led to patients with Alzheimer's disease [18]. The cell operations can help to gather an idea regarding how to dedicate healthcare service to the patient and fault detection in IoMT in a timely manner, due to the complexity of the safe relationship between layers.

The mentioned studies point to the problems with IoMT and exciting discussions about the issue, including:

- Rising the complexity of the relationship between different components in IoMT layers;

- Deploying the Internet of Medical Things and dataflow balancing between its nodes;

- Increasing the probability of occurring the faults and becoming defective devices;

- Detecting defective devices before failure the dependable communication between components;

- The highlighted role of the inspired approaches from the human brain's neuron cell structure to support the complexity.

According to the role of the brain neuron cell structure and support of all mechanisms in the human body with high complexity, we review the astrocytes' operations to distribute the cholesterol into the neurons and tackle cellular waste by phagocytosis. After analyzing the Alzheimer's internal symptoms and astrocyte operations, we propose an astrocyte flow-mapping method to distribute the traffic of IoMT-based applications onto a mesh network's PEs and detect defective devices. To follow the issue, this work defines a task graph of vaccination against COVID-19 and maps it onto mesh nodes, according to the network characteristics in distributing traffic in four directions and deadlock-free routing algorithms [19-23]. The features create an opportunity to distribute the traffic based on astrocyte cell and phagocytosis operations and cluster the mesh nodes by inspiring the cell structure. We propose a model to classify the processing elements into similar categories for balancing the flow between nodes and quickly detecting defective PEs by evaluating 
the mapped node's performance, which is located on a cluster. The case study describes mapping, clustering, and detecting defective devices by presenting the equations. A defective node is detected in a timely manner before failure due to a dependable relationship between the mapped tasks and investigating the processing elements in the different clusters with fewer PEs than the total number presented. We collect a dataset from vaccination COVID-19 and present an algorithm for astrocyte-flow mapping and detecting defective devices (AFMD), which define a task graph including a vaccine distribution center, a vaccination center, and an emergency center. AFMD maps the tasks and distributes their traffic onto a mesh that the proposed algorithm employs the real collected dataset of vaccination COVID-19 to follow the purposes. After detecting the defective devices and pruning them, the algorithm re-maps the tasks onto mesh PEs due to the updated acknowledgments of the available network nodes. This work passes the different steps necessary to satisfy its purposes, as shown in Figure 1.

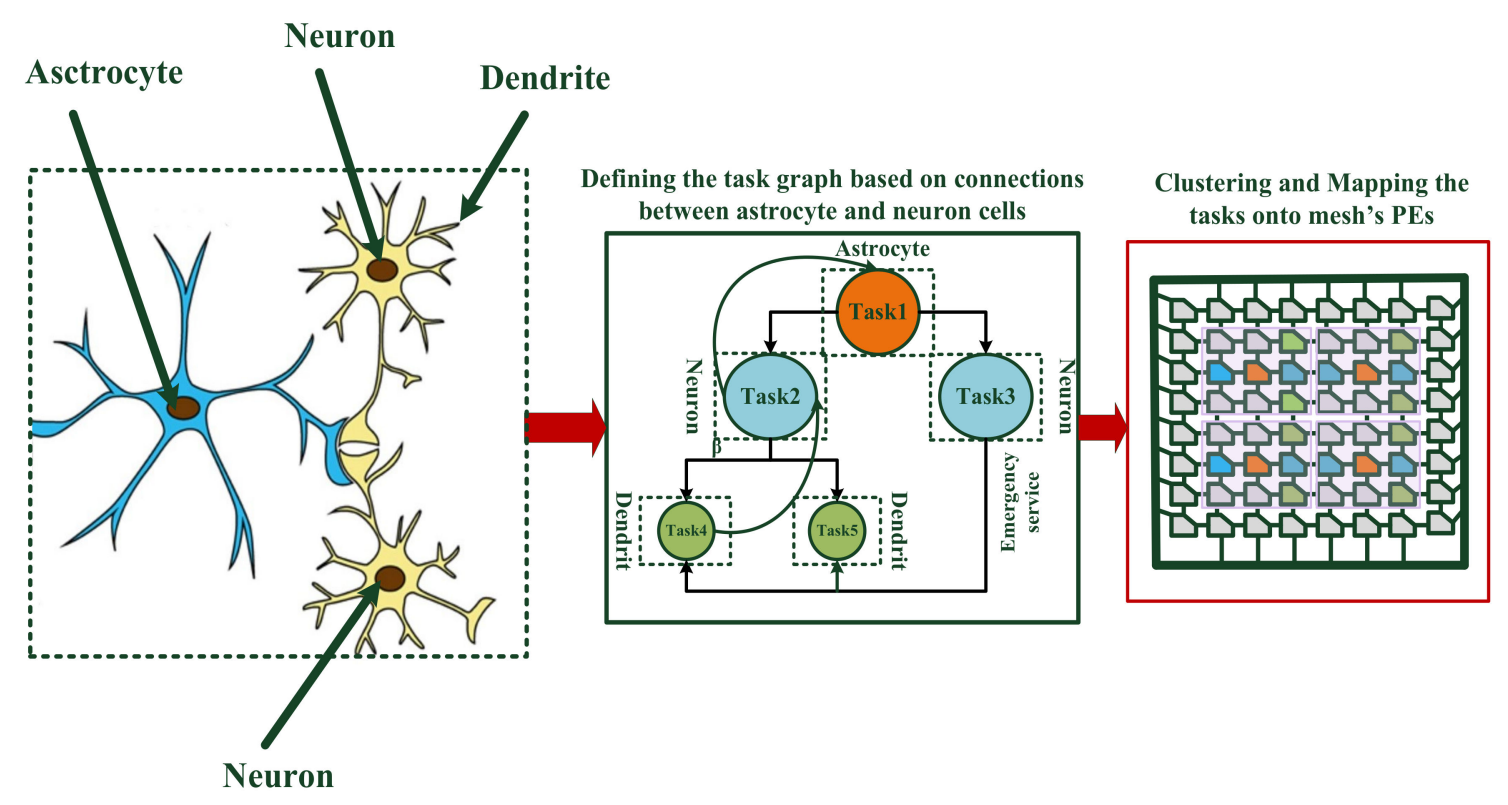

Figure 1. The stages of the proposed idea.

By reviewing the upper concepts and the pointed IoMT challenges, we point the study's main contributions and novelties as follows:

- Inspiring the astrocyte cell operation to fairness distribute IoMT traffic onto mesh nodes;

- Detecting defective devices based on astrocyte and phagocytosis operation to tackle increasing the probability of failure communication between IoMT components;

- Defining a task graph of the COVID-19 vaccination based on the relationship between astrocytes, neurons, and dendrites;

- Presenting an algorithm and a model to map the tasks onto a mesh network;

- Describing AFM, clustering the network, and detecting a defective node by presenting equations.

In reviewing the main contribution and novelty, we employ the astrocyte pattern to fairly distribute cholesterol to neurons and nourish them for mapping a task graph onto the mesh network nodes and balancing flow onto their processing elements. The study defines a task graph based on an IoMT application and addresses the relationship between vaccination distribution, vaccine, and emergency centers as one of the highlighted applications of the Internet of Medical Things due to the COVID-19 epidemic. We also inspire the phagocytes operations to eliminate waste for detecting defective devices and removing them from the application nodes, which are mapped onto the network's PEs. 
After timely detecting fault, IoMT nodes are re-mapped onto the mesh processing elements and transferred data between them by updating the initial information for AFMD.

To evaluate the study's role in improving the Internet of Medical Things, we compare the reported communication delay and energy consumption after and before performing AFMD and injecting random fault with different rates onto the IoMT components in different layers, including physical, information integration, and application service. This work estimates the performance of astrocyte-defective device detection before and after injecting random faults and pruning the failed processing element. The timely detection of the defective devices and balancing dataflow onto mesh processing elements leads to improve the idea efficiency in increasing the performance of IoMT-based applications.

The next sections of the case study follow the issues that are included: Section 2 reviews the related researches to the provided approaches for improving IoMT performance from different angles; the astrocyte-flow mapping, AFMD, and equations are explained in Section 3; Section 4 evaluates the reported results by the simulators to demonstrate the idea efficiency in facing the challenge of IoMT; Section 5 discusses the presented observations and simulation results; and a conclusion of the background and purposes the work is presented in Section 5.

\section{Related Work}

Many case studies addressed IoMT's weaknesses and advantages to provide the solutions against the challenges improve the performance and cost of the Internet of Medical Things. Some researchers utilized the human neural structures to simulate and implement different multi-layer systems with high complexity. The Internet of Medical Things followed the multi-layer structures in that its deploying increased the complexity of the relationship between its nodes and computational operations. This section reviews the related works to the mentioned concepts, according to the study's research area.

The researchers almost focused on detecting the active and passive attacks on the Internet of Medical Things due to sharing the patient records and the probability of intruders' access to their private information. Kumar et al. [24], detected the cyber-attacks in fog and cloud layers that have employed the machine learning algorithms, including decision tree and random forest. The study proposed architecture to support the heterogeneous communication infrastructures (Such as IoMT), which could detect the attacks with high accuracy by providing a structure, including software and infrastructure as services (SaaS and IaaS) in fog and cloud layers (Integration information and application layers in IoMT). Reducing the number of cyber-attacks affected facing them and satisfying the security and quality of service besides presenting attack detection methods, in which the researchers followed the issue by identifying the malicious attacks, vulnerabilities, and consensus of hybrid multi-agent systems with malicious nodes via security and authentication protocols [25]. Khan et al. [26] also addressed malicious malware detection by presenting a software-defined network (SDN) framework and deep learning approach to timely decisions about malware with high precision and accuracy without imposing cost overhead. The different learning techniques had a highlighted role in detecting the faults and deciding about them in a timely manner to satisfy the security in IoT and IoMT such as reinforcement learning to online training and diagnosis of the cyber-attacks [27].

Verifying the signature affects provision of the fault tolerance-based methods to protect the shared records tackling the intruders and different attacks. The researchers addressed the different protocols to identify an invalid signature after testing based on group technologies, including bitcoin, a combined framework of different security protocols, and Hyperledger Fabric [28]. The case studies utilized the sensor ID, signature, and a shared key to verify the security and the sensor validation that have supported the trust in different layers of IoMT, according to restrictions of the main contribution of security in integration information and application layers. Das et al. [29] focused on increasing the fault tolerance to satisfy the security in distributing the COVID-19 vaccine using blockchain framework, according to its features, including transparency, decentralization, and immutability. The 
work utilized artificial intelligence ( $\mathrm{Al}$ ) to monitor and control the related data to vaccination that consists of request, supply, and the entered dose of vaccine. The blockchain provided a secure framework that employing Al-based methods to protect privacy and improve fault tolerance satisfied the security of the IoMT application. According to the lack of storing the data transaction between IoMT nodes in the blockchain, the researchers utilized an artificial neural network and machine learning algorithm to predict the different attacks and protect the information security.

Some case studies addressed the Internet of Medical Things to detect the related diseases to the brain (Such as Epilepsy, Brain tumor, Parkinson) by employing fuzzy-based approaches to analyze the reported wearable and biomarker sensors' information [30,31]. Khan et al. [32], detected the brain tumor that timely diagnosed and predict the grade of the disease by analyzing the details of the condition of brain cells with a partial tree. Deng et al. [33], also graded the brain tumor using the heterogeneous convolutional neural networks to medical image processing, in which the study employed IoMT applications to share the patient records and timely dedicate suitable healthcare service providers to the patients with the disease.

The brain neural structure affects facing the complexity in different communications between components that astrocyte has a highlighted role in the nourishing the neuron cells. Liu et al. [34] proposed HANA that was inspired the astrocyte distribution pattern to provide the architecture for reducing the communication between the nodes of an NoCbased infrastructure. The researchers tiled the network based on the astrocyte structure and analyzed the performance of HANA due to the number of cells that have presented an application-specific integrated (ASIC) design. Indeed, HANA has provided an ASICdesign that has only supported applications with a similar pattern to astrocyte cells. Our idea utilizes the pattern of the relationship between astrocytes, neurons, and dendrite to map the tasks and distribute their traffic onto the mesh network's PEs. Since we propose a flexible structure to support different applications with high efficiency without changing tiling techniques in a mesh topology. Table 1 indicates an overview of the mentioned studies, which consists of their methods, advantages, and weaknesses.

Table 1. An overview of the mentioned studies.

\begin{tabular}{|c|c|c|c|}
\hline Case Study & Method & Advantage & Weakness \\
\hline Kumar et al. [24] & $\begin{array}{l}\text {-Detecting attacks } \\
\text {-SaaS and IaaS } \\
\text {-Machine learning algorithms }\end{array}$ & $\begin{array}{l}\text {-Detecting attacks in fog and } \\
\text { cloud layers of IoT and IoMT } \\
\text {-Satisfying IoMT security }\end{array}$ & $\begin{array}{l}\text {-Only secure-based service provider } \\
\text {-Focusing two layers } \\
\text {-Lack of solution for faulty components }\end{array}$ \\
\hline Rahman et al. [25] & $\begin{array}{l}\text {-Detecting cyber-attacks } \\
\text {-Detecting vulnerabilities } \\
\text {-Authentication protocols }\end{array}$ & $\begin{array}{l}\text {-Supporting cyber-attacks } \\
\text { and vulnerabilities } \\
\text {-Satisfying IoMT security }\end{array}$ & $\begin{array}{l}\text {-Only secure-based service provider } \\
\text {-Lack of solution for faulty components }\end{array}$ \\
\hline Khan et al. [26] & $\begin{array}{l}\text {-Detecting malicious malware } \\
\text {-SDN } \\
\text {-Deep learning }\end{array}$ & $\begin{array}{l}\text {-Timely detecting malware } \\
\text {-High precision and accuracy }\end{array}$ & $\begin{array}{l}\text {-Only secure-based service provider } \\
\text {-Lack of solution for faulty components }\end{array}$ \\
\hline $\begin{array}{l}\text { Chaabouni et al. } \\
\text { [27] }\end{array}$ & $\begin{array}{l}\text {-Detecting cyber-attacks } \\
\text {-Reinforcement learning }\end{array}$ & -Timely detecting attacks & $\begin{array}{l}\text {-Only secure-based service provider } \\
\text {-Lack of solution for faulty components }\end{array}$ \\
\hline Xiong et al. [28] & $\begin{array}{l}\text {-Verifying signature } \\
\text {-Bitcoin } \\
\text {-Security protocols }\end{array}$ & $\begin{array}{l}\text {-Satisfying trust of IoMT } \\
\text {-Supporting different layers }\end{array}$ & $\begin{array}{l}\text {-Trust-based service provider } \\
\text {-Lack of solution for faulty components }\end{array}$ \\
\hline Das et al. [29] & $\begin{array}{l}\text {-Blockchain } \\
\text {-Artificial neural network } \\
\text {-Machine learning algorithm }\end{array}$ & $\begin{array}{l}\text {-Satisfying IoMT security } \\
\text {-Fault tolerance }\end{array}$ & $\begin{array}{l}\text {-Only secure-based service provider } \\
\text {-Lack of solution for faulty components }\end{array}$ \\
\hline Kumar et al. [30] & $\begin{array}{l}\text {-Diagnosing infectious } \\
\text { diseases } \\
\text {-Numerical models } \\
\text {-Fuzzy-based approach }\end{array}$ & $\begin{array}{l}\text {-Diagnosing infectious } \\
\text { diseases } \\
\text {-Predicting infectious diseases }\end{array}$ & $\begin{array}{l}\text {-Approximation model } \\
\text {-Hidden the fault role }\end{array}$ \\
\hline
\end{tabular}


Table 1. Cont.

\begin{tabular}{|c|c|c|c|}
\hline Case Study & Method & Advantage & Weakness \\
\hline Wang et al. [31] & $\begin{array}{l}\text {-Similarity measurement } \\
\text {-Fuzzy-based approach }\end{array}$ & $\begin{array}{l}\text {-Finding similarities with } \\
\text { high accuracy }\end{array}$ & $\begin{array}{l}\text {-Approximation model } \\
\text {-Hidden the fault role }\end{array}$ \\
\hline Khan et al. [32] & $\begin{array}{l}\text {-Detecting brain tumor } \\
\text {-Predicting grade of } \\
\text { brain tumor } \\
\text {-Partial tree }\end{array}$ & $\begin{array}{l}\text {-Timely detecting brain tumor } \\
\text {-Predicting grade of brain } \\
\text { tumor with high accuracy } \\
\text {-Employing IoMT }\end{array}$ & $\begin{array}{l}\text {-Lack of Supporting rising computational } \\
\text { complexity and IoMT challenges }\end{array}$ \\
\hline Deng et al. [33] & $\begin{array}{l}\text {-Detecting brain tumor } \\
\text {-Predicting grade of } \\
\text { brain tumor } \\
\text {-Convolutional neural } \\
\text { network } \\
\text {-Medical image processing }\end{array}$ & $\begin{array}{l}\text {-Timely detecting brain tumor } \\
\text {-Predicting grade of brain } \\
\text { tumor with high accuracy } \\
\text {-Employing IoMT } \\
\text {-Timely providing } \\
\text { healthcare service }\end{array}$ & $\begin{array}{l}\text {-Lack of Supporting rising computational } \\
\text { complexity and IoMT challenges }\end{array}$ \\
\hline Liu et al. [34] & $\begin{array}{l}\text {-Network on chip } \\
\text {-Tiling technic } \\
\text {-Astrocyte cells pattern }\end{array}$ & $\begin{array}{l}\text {-Tiled the network based on } \\
\text { the astrocyte structure } \\
\text {-Reducing latency and } \\
\text { energy consumption }\end{array}$ & $\begin{array}{l}\text {-ASIC-based design } \\
\text {-Low flexibility } \\
\text {-Low scalability }\end{array}$ \\
\hline This study & $\begin{array}{l}\text {-Providing astrocyte-mapping } \\
\text { flow algorithm } \\
\text {-Clustering mesh nodes } \\
\text {-Providing IoMT } \\
\text { flow mapping }\end{array}$ & $\begin{array}{l}\text {-Timely detecting fault } \\
\text {-Reducing total runtime } \\
\text { and energy } \\
\text {-High flexibility and scalability } \\
\text {-Balancing flow into IoMT } \\
\text { applications' nodes }\end{array}$ & -Traffic pattern complexity \\
\hline
\end{tabular}

According to the mentioned studies as the related works, the flow balancing and timely fault detection are challenging for the IoMT infrastructures, with a rising complexity of the relationship between their nodes and computational operation. Many studies focused on the issues to present attack detection, fault tolerance, and trust definition to satisfy the security on the Internet of Medical Things. Additionally, the reviewed works demonstrate the role of machine learning algorithms and fuzzy-based approaches to tackle the challenges. Nevertheless, the researchers employed IoMT for timely detecting and predicting the brain's diseases and almost addressed identifying the attacks as the parameters that could be deteriorated the security; however, the role of hardware-based failures is invisible. Therefore, we focus on timely detecting hardware-based defective devices and flow balancing between IoMT nodes in different layers to reduce its complexity by employing an astrocyte cholesterol distribution pattern to map the IoMT application's tasks and traffic.

\section{Astrocyte-Flow Mapping and Defective Device Detecting}

This section provides the details of our work steps to map the tasks onto mesh processing elements and detect a failed or defective node after distributing an IoMT application traffic. To satisfy the purposes, we define a task graph based on an application of the Internet of Medical Things for analyzing AFMD's efficiency. The section explains collecting a dataset and distributing its traffic onto the network's PEs, according to the defined task graph and astrocyte cell operation. Additionally, the proposed algorithm is explained to demonstrate the role of the clustering method and detecting defective devices that are inspired by phagocytosis operation.

\subsection{Task Graph and Data Collection}

After investigating the astrocyte cell operation and its relationship to the neurons for nourishing them, we define a task graph, in which the relationship between tasks imitates the cell pattern. The study focuses on IoMT- based application to present a task graph to prove AFMD's role in improving its efficiency, which consists of the vaccine distribution, 
vaccination, emergency centers, and clients, as shown in Figure 2. The centers of vaccine distribution, vaccination, emergency, and clients have inspired the astrocyte, neuron, and dendrite, respectively. According to the task graph, the vaccine distribution center provides a service to the emergency center besides assigning service to the vaccination center for critical situations and occurring disruption in a client's vital condition. The received correct or incorrect feedback of a client about her health situation affects dedicating a correct service from the vaccination center and final result.

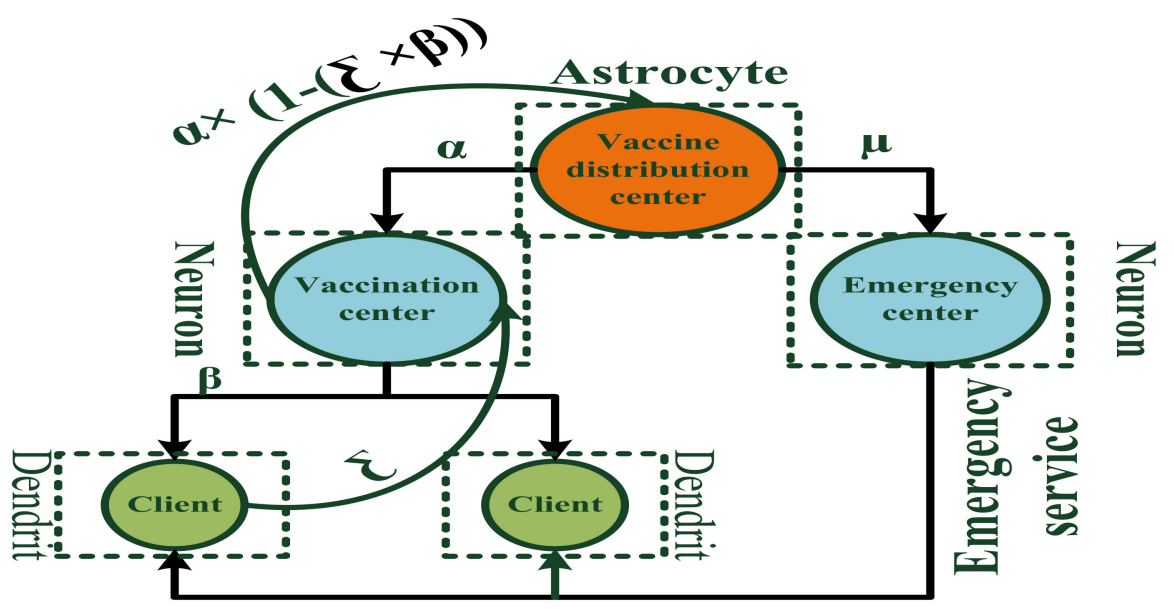

Figure 2. The defined task graph based on an IoMT application and the relationship between astrocyte, neuron, and dendrite.

To compute the probability of dedicating the healthcare service provider (Emergency service), we estimate the probability of occurring a mistake decision or feedback from the vaccination center and client, as shown in Equation (1). In Table 1, we describe the existing parameters of the task graph to compute the probability of dedicating an emergency service to the client.

$$
\text { Emergency }_{\text {service }}=\mu \times \frac{1}{\alpha \times(1-(\varepsilon \times \beta))}
$$

The study investigates the registered information in laboratory monitoring the astrocyte operation to find a pattern for distributing traffic onto mesh nodes and utilizes the real dataset of vaccination COVID-19 to describe traffic traces. Since we collect a dataset, which includes the registered information about astrocyte cell operations and vaccination, and share it on Github (https: / / github.com/yasamanhosseini/ Astrocyte-flow-mappingproject accessed on 8 October 2021) [35-37].

To present a fair report of the idea efficiency, this work simulates the defined tasks as the healthcare service provider nodes and sensors and injects fault (based on a random rate) to them using the CupCarbon simulator tool [38].

Figure 3 demonstrates that the traffic traces are defined based on the collect dataset, including vaccination COVID-19 and a pattern of astrocyte operations. 


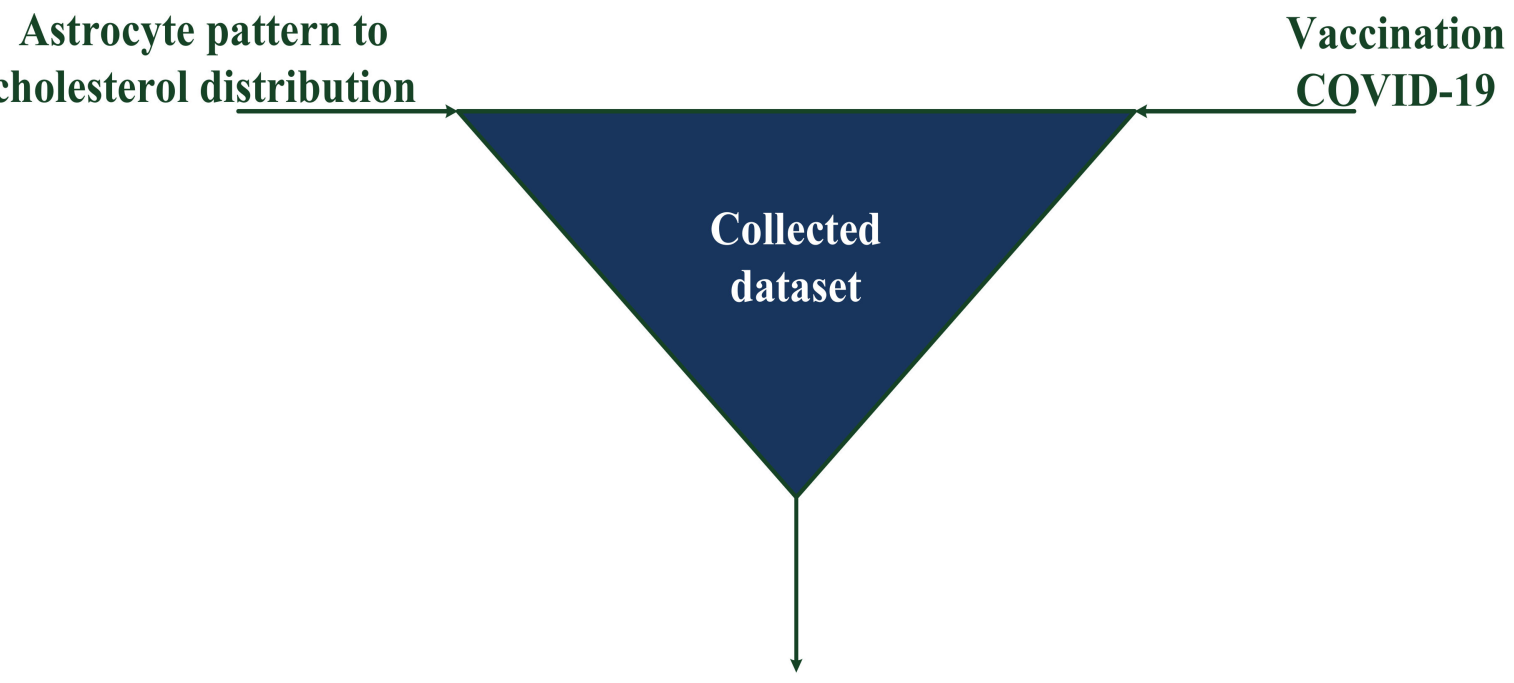

\section{Traffic traces for mapping on mesh nodes}

Figure 3. The collected dataset, including astrocyte pattern to cholesterol distribution into the neurons and vaccination COVID-19.

\subsection{Equation and Astrocyte-Flow Mapping Algorithm}

The subsection is aimed at the details of the provided equations and proposed algorithm to explain mapping the tasks' dataflow onto a mesh network-processing element and the equations.

We define a mesh topology as a binary matrix (M), in which Equation (2) describes astrocyte mapping as task 1 (Vaccine distribution center). Table 2 illustrates the parameters that are employed to define the equations.

Table 2. Explaining the employed parameters in the defined equations.

\begin{tabular}{|c|c|c|}
\hline PARAMETER & DESCRIPTION & CONDITION \\
\hline A & The probability of providing service to the vaccination center & $0 \leq \alpha \leq 1$ \\
\hline $\mathrm{B}$ & The probability of providing service to a client & $0 \leq \beta \leq 1$ \\
\hline$\mu$ & The probability of providing service to the Emergency center & $0 \leq \mu \leq 1$ \\
\hline$\varepsilon$ & The probability of the client awareness from her situation & $0 \leq \varepsilon \leq 1$ \\
\hline M & Mesh network's nodes & - \\
\hline A & Astrocyte cell & - \\
\hline $\mathrm{C}$ & The number of clusters & $i \geq 1$ \\
\hline I & The number of mesh network's row & $i \bar{i} \geq 0$ \\
\hline $\mathrm{J}$ & The number of mesh network's column & $\mathrm{j} \geq 0$ \\
\hline $\mathrm{M}_{\mathrm{I}, \mathrm{J}}$ & A matrix's element in location's row $i$ and column $j$ & $a_{i, j}=1$ \\
\hline$N$ & The number of astrocyte cell & $\mathrm{n} \geq 0$ \\
\hline $\mathrm{I}_{\mathrm{MAX}}$ & The maximum number of a mesh network's rows & $i_{\max } \geq 0$ \\
\hline $\mathrm{J}_{\mathrm{MAX}}$ & The maximum number of a mesh network's column & $\mathrm{j}_{\max } \geq 0$ \\
\hline SUBCLUSTER $_{\text {NUMBER }}$ & The sub-cluster number & - \\
\hline PROCESSING ELEMENT NUMBER $_{\text {NUM }}$ & The number of the located processing element into each cluster & - \\
\hline FAULT & $\begin{array}{l}\text { Receive a faulty acknowledgment from the mapped neuron at } \\
\text { t moment }\end{array}$ & $0 \leq$ Fault $\leq 1$ \\
\hline $\mathrm{T}$ & Available time & $t \geq 0$ \\
\hline $\mathrm{T}_{\mathrm{MAX}}$ & Maximum available time & $t_{\max } \geq 0$ \\
\hline DEFECTIVE DEVICE & Detect a defective device based on occurring fault & $0 \leq$ Defective device $\leq 1$ \\
\hline $\mathrm{AM}$ & Astrocyte mapping (vaccine distribution center) & - \\
\hline NM & Neuron mapping (vaccination and emergency centers) & \\
\hline
\end{tabular}




$$
\begin{array}{r}
M=\left[\begin{array}{ccc}
m_{0,0} & \cdots & m_{0, j} \\
\vdots & \cdots & \vdots \\
m_{i, 0} & \cdots & m_{i, j}
\end{array}\right] \\
A M=\left\{\begin{array}{r}
m_{((2 \times i)+2), j+2}=A_{n},\left(1 \leq i<\left\lceil\frac{i_{\max -4}}{2}\right\rceil\right) \wedge\left(1 \leq j<\left\lceil\frac{j_{\max -4}}{2}\right\rceil\right) \wedge\left(0 \leq n<\frac{n_{\max }}{2}\right) \\
m_{((2 \times i)+1),((2 \times i)+2)}=A_{n},\left(\left\lceil\frac{i_{\max }-4}{2}\right\rceil \leq i<\left(i_{\max }-4\right)\right) \wedge\left(\left\lceil\frac{j_{\max -4}}{2}\right\rceil \leq j<\left(j_{\max }-4\right)\right) \wedge\left(\frac{n_{\max }}{2} \leq n \leq n_{\max }\right)
\end{array}\right.
\end{array}
$$

Equation (3) describes mapping the neuron cells based on the pattern of the relationship between astrocytes and the cells that their role is defined as the emergency and vaccination centers, according to the task graph.

$$
N M=\left\{\begin{array}{c}
m_{((2 \times i)+2),((2 \times j)-1)}=N_{o},\left(1 \leq i \leq\left\lceil\frac{i_{\max -4}}{2}\right\rceil\right) \wedge\left(1 \leq j \leq\left\lceil\frac{j_{\max -3}}{2}\right\rceil\right) \wedge\left(0 \leq o<\frac{o_{\max }}{2}\right) \\
m_{((2 \times i)+1),((2 \times j)-1)}=N_{o},\left(\left\lceil\frac{i_{\max }-4}{2}\right\rceil<i \leq\left(i_{\max }-4\right)\right) \wedge\left(\left\lceil\frac{j_{\max -3}}{2}\right\rceil<j \leq\left(j_{\max }-3\right)\right) \wedge\left(\frac{o_{\max }}{2} \leq o \leq o_{\max }\right)
\end{array}\right.
$$

This work presents a clustering method to categorize the mesh nodes with inspiring the relationship and communication pattern between astrocyte, neuron, and dendrite. Before clustering, we determine the total number of processing elements that are located

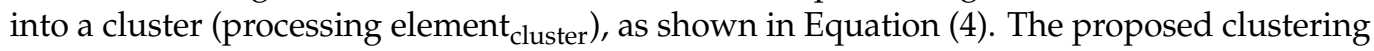
method is provided by considering the network structure and feature besides analyzing the communication pattern between the mentioned brain cells and dendrites.

$$
(\text { Processing element })_{\text {cluster }}=\frac{1}{4} \times \sum_{\substack{j=j_{\max }-1 \\ i=i_{\max }-1 \\ i=1}}^{\substack{i=1 \\ j=1}}
$$

Theorem 1. $m_{i, j}$ is an element of binary matrix $[M]_{i, j}$, which is located on $i$ row and $j$ column of $[M]_{i, j}$ in Equation (4).

Example 1. Verifies Equation (4) double summation operation where $1 \leq i \leq 3$ and $1 \leq j \leq 3$ are met.

$$
(\text { Processing element })_{\text {cluster }}=\frac{1}{4} \times \sum_{\substack{j=2 \\ i=1 \\ j=1}}^{\substack{i=2 \\ j=1}} m_{i, j}=\frac{1}{4} \times\left(\sum_{\substack{i=2 \\ j=1}}^{j=2} \sum_{\substack{i=1 \\ j=1}}^{j=2} m_{i, j}\right)=\frac{1}{4} \times\left(\left(m_{1,1}+m_{1,2}\right)+\left(m_{2,1}+m_{2,2}\right)\right)
$$

After determining the number of clusters based on the total number of the located PEs into a cluster, Equation (5) defines our clustering method where c demonstrates the cluster number.

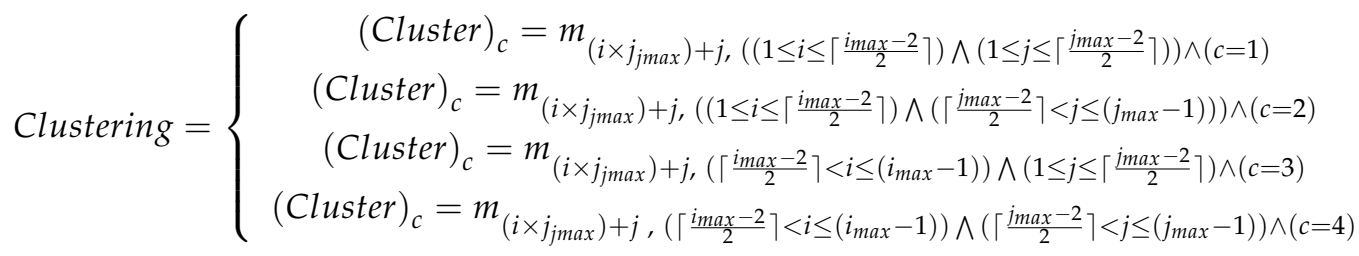


According to astrocyte structure and connection between it and neurons, we classify each cluster into sub-clusters where (total number of the located PEs into a cluster) $\geq 18$ is met. The proposed sub-clustering method is described by Equation (6).

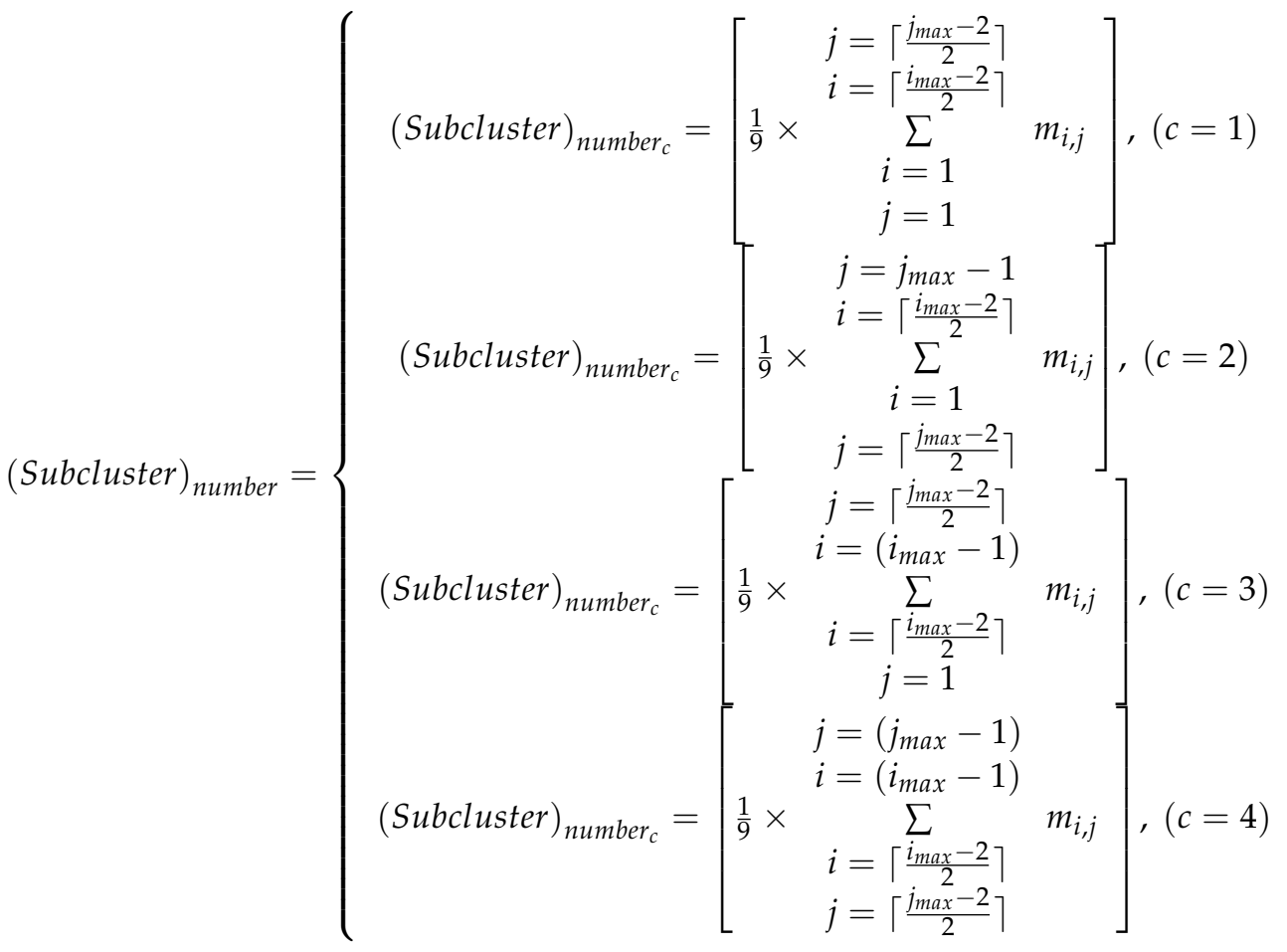

The highlighted purpose of the study addresses timely detecting a defective device in an IoMT application because of rising complexity in the communication between its components and computational operations. Therefore, we propose a method to detect a defective node (as the mapped node by a task) based on phagocytosis operation against waste cells, as shown in Equation (7).

$$
(\text { Defective device })_{i, j}=1-\frac{\sum_{t=0}^{t=t_{\max }}(\text { fault })_{\left(m_{i, j}\right)_{t}}}{t_{\max }}
$$

After presenting the mentioned equations to describe mapping, clustering, and detecting a defective device, the algorithm demonstrates the steps of implementing operation and clarifies the proposed method to timely fault detection. Algorithm 1 demonstrates AFMD steps to satisfy the work's purposes and explains the existing parameters and conditions in the algorithm. More AFMD's time complexity is related to mapping the defined tasks onto the mesh nodes and distributing their traffic onto its PEs. We explain the mentioned operations and the updated initial information after detecting fault by describing the traffic traces and imposing them on the simulator, which reports total runtime and energy consumption. 
Algorithm 1. Astrocytes-flow and defective device detection (AFMD) (An astrocyte-flow mapping and defective device detecting (AFMD) algorithm)

Parameter deceleration:

$\mathrm{T}:$ Task $=($ Vaccine distribution center. Vaccination center. Emergency center, Client $)$

i: Task index $=\{0,1,2,3\}$

$\mathrm{j}$ : Total number task index whereas $q \subset$ Natural number and $j \geq 0$

q: Mesh's row index whereas q $\subset$ Natural number and $q \geq 0$

p: Mesh's column index whereas $p \subset$ Natural number and $p \geq 0$

$\mathrm{t}$ : Available time whereas $\mathrm{t} \subset$ Natural number and $\mathrm{t} \geq 0$

$a_{\text {index note: }}$ A matrix element whereas a is a binary matrix

$\mathrm{x}$ : Mesh's cow index whereas $\mathrm{x} \subset$ Natural number and $\mathrm{x} \geq 0$

Inputs: 1- DF: Data-flow of the task graph (Vaccine distribution center, Vaccination center, Emergency center, Client) whereas SV $\subset$ Natural number

2- DetectedFault: The detected fault on a mesh's processing element as the mapped neuron

whereas $0 \leq$ DetectedFault $\leq 1$

Output: DefectiveDevice: The detected defective devices (The mapped neurons) whereas $0 \leq$ DefectiveDevice $\leq 1$

1: Start

2: Procedure CAS (DF.DetectedFault, DefectiveDevice)

3: Start phasel // Task mapping (Astrocytes-flow mapping) onto mesh's processing element

4: While each $\mathbf{t}$ in Available time do

5: $\quad$ for each $i$ in Task index do

6: $\quad$ for each $q$ in Mesh's row index do // Mapping the task on mesh nodes

7: $\quad \mid$ for each $p$ in Mesh's column index do

8: $\quad \mid$ for each $\mathbf{j}$ in Total number task index do

9: $\quad \mid($ IndexNode $) \leftarrow\left(q \times p_{\max }\right)+p$

10:

11:

$(a)_{t_{\text {indexnote }}} \leftarrow D F_{i_{j t}}$

Defective device $_{\text {Index } \mathrm{Note}} \leftarrow 1-\quad \frac{\sum_{t=0}^{\mathrm{t}=t_{\max }} \text { (DetectedFault) }_{\text {IndexNote }}}{t_{\max }}$

12:

13:

14:

15:

16:

17:

18:

19:

20:

21:

22:

23:

24: end process;

25: end 
AFMD first maps the defined tasks (vaccine distribution, vaccination, and emergency centers and clients) onto the network's processing elements, according to the presented equations to follow the issues. The algorithm searches the occurring fault on the processing elements in the separate determined clusters that are led to detect a fault faster than investigating the situation of different nodes in a mesh topology. We define a threshold value to identify a node as a defective device and prune it from the network. This value is determined by evaluating the processing elements' efficiency and their correct data transaction rate. AFMD detects a defective device, prunes it and updates the information about the network where (Defective device) IndexNode $<$ (Threshold value) is met. After detecting the defective nodes in a period $\left(t=t_{\max }\right)$, the failed PEs are pruned and re-mapped the tasks onto the network nodes based on the updated initial information of the topology, which the concept is clearly explained in the next subsection.

\subsection{Astrocyte-Flow Mapping Onto Mesh Network Nodes}

According to the special and successful operation of astrocyte cells to distribute cholesterol into the neurons and its phagocytosis against the waste cells, the work utilizes the astrocyte pattern to IoMT traffic distribution onto mesh network's PEs and fault detection onto them. Nevertheless, the type of network-on-chip (NoC) based communication infrastructure, traffic pattern, flow mapping and data or bus scheduling method, and clustering the network nodes affect improving or deteriorating the ideal performance $[19,20,39]$.

We utilize a mesh network to distribute the traffic in four directions and multicasting it onto the network processing elements and clustering its nodes based on astrocyte pattern and relationship between tasks. Additionally, the study classifies the clusters to the subcluster where (Total number of the located PEs into a cluster) $\geq 18$ is met. Figure $4 a-c$ demonstrate clustering, sub-clustering, and clustering where (total number of the located PEs into a cluster) $<18$ is met, respectively.

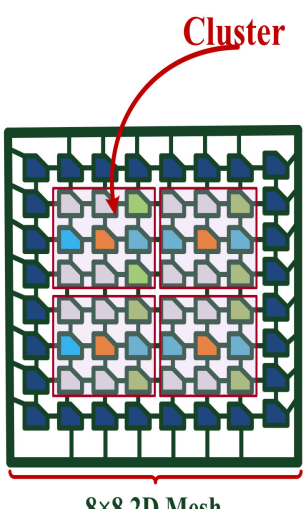

(a)

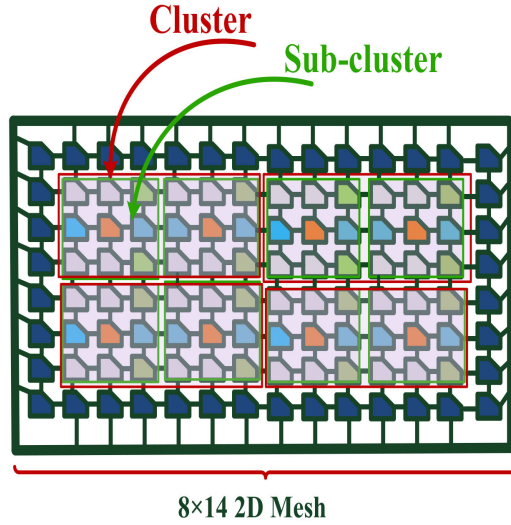

(b)

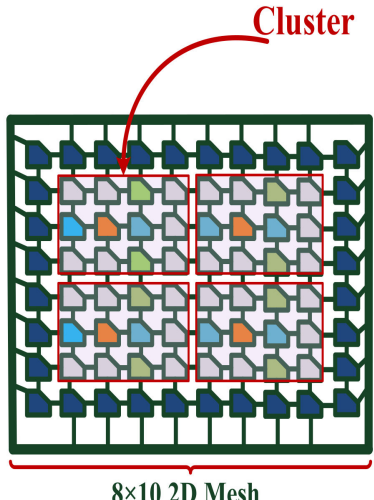

(c)

Distributer node

Figure 4. The proposed clustering and sub-clustering methods; (a) clustering the mapped nodes based on the relationship between tasks; (b) defining the sub-clusters into main four clusters; (c) clustering and sub-clustering ((total number of the located PEs into a cluster) $<18)$.

After clustering and mapping the tasks onto the processing element, this work analyzes the performance of the nodes in the separate cluster to detect fault and decisions about determining a defective device. We face the defective devices based on two assumptions, including pruning them from the topology (Assumption 1) and considering them as circuit switching (Assumption 2). To tackle assumption 1 that inspire from phagocytosis operation, AFMD re-maps the network's processing units based on the updated information about the topology, including the pruned nodes from a mesh. Figure $5 \mathrm{a}, \mathrm{b}$ show mapping the tasks 
onto $8 \times 8$ 2D Mesh before detecting defective node and re-mapping them onto the nodes after detecting defective PEs and pruning them (Assumption 1). In assumption 2, we utilize the feature of circuit switching, which removes the probability of occurring the related fault to short circuit and open circuit, as shown in Figure 5c. The study demonstrates reducing the total hopcounts per cluster for Assumption 2, compared with assumption 1 when the algorithm detects the defective devices.

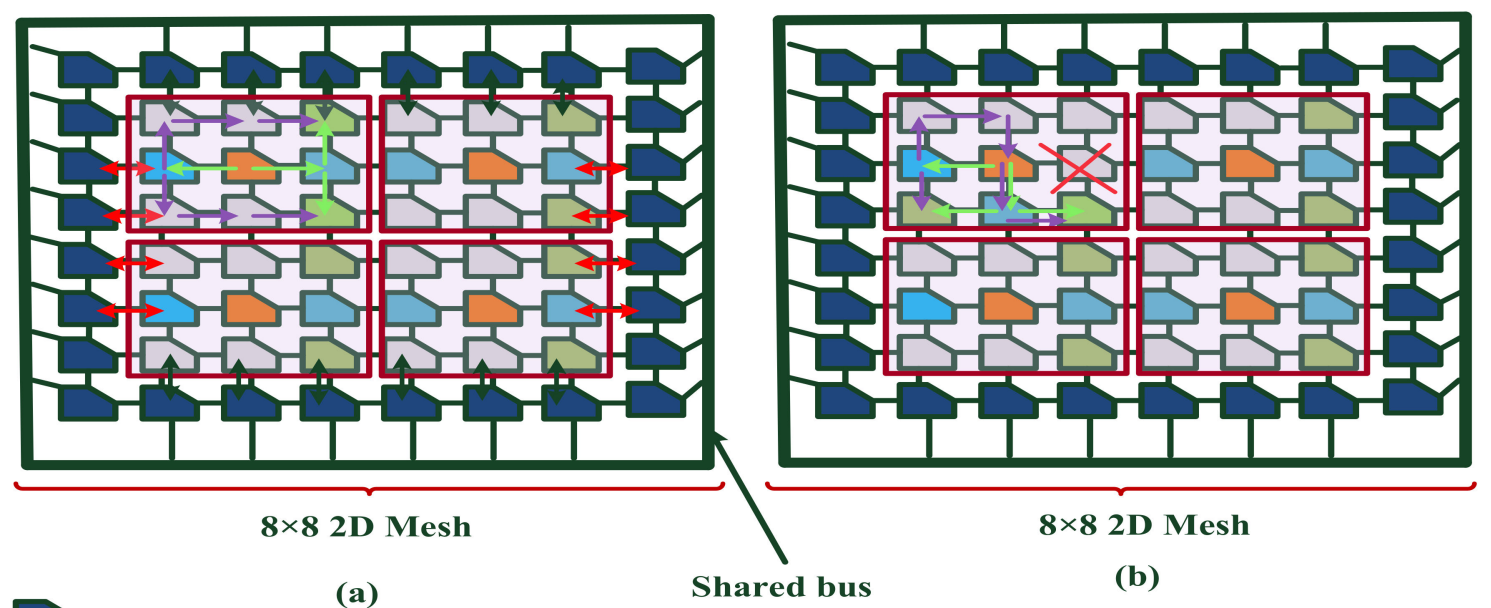

Distributer node

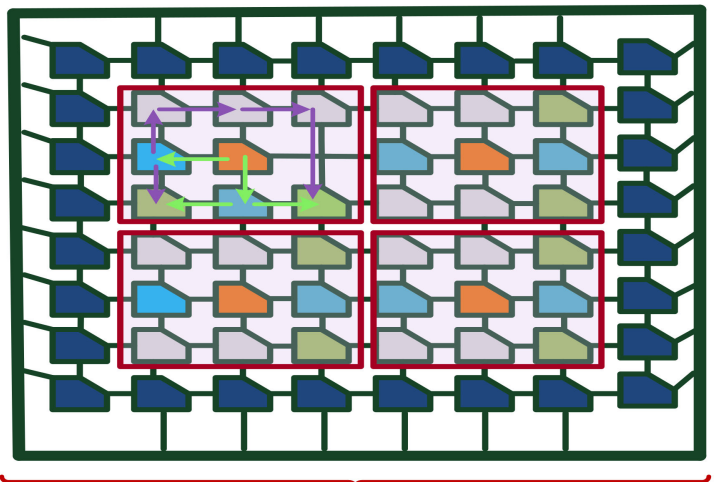

8×8 2D Mesh

(c)

Figure 5. The data transfer between the mapped tasks onto $8 \times 8$ 2D Mesh PEs before and after detecting a defective node; (a) mapping the tasks onto $8 \times 8$ 2D Mesh PEs before detecting a defective node (the black-color pointer demonstrate the data transfer between the located nodes into up and down partitions with the connected distributer nodes to the shared bud); (b) re-mapping the tasks onto $8 \times 8$ 2D mesh PEs before detecting a defective node (vaccination center); (c) pruning the defective node (vaccination center) and re-mapping the tasks onto $8 \times 82 \mathrm{D}$ Mesh PEs.

To describe our method for distributing the IoMT application traffic onto the network's PEs, we distribute the traffic onto the mesh nodes from the four directions of left, right, up, and down. The black-color pointers demonstrate data transfer between up and down partitions with the connected distributer nodes to the shared bus, in which the red-color pointers illustrate data transfer between left, right partitions, and the distributer nodes.

By analyzing the mentioned concepts in the section, employing an astrocyte pattern to distribute cholesterol into the neuron affects supporting the complexity of the relationship between IoMT nodes and layers. We defined a task graph based on the relationship between astrocyte, neuron, and dendrite, which follows an IoMT application to evaluate the idea's efficiency in improving the Internet of Medical Things to support communication between its nodes and tackle defective devices after failure more nodes. The issue has a highlighted role to provide dependable healthcare service to the patients with reducing the 
probability of occurring a risky condition for the requesters. The algorithm can help to map the different tasks (Various IoMT applications) onto the different NoC-based infrastructures and detect defective nodes before the failure of the system.

\section{Simulation Results}

We simulate the mapped tasks onto mesh processing elements and the IoMT application's traffic distribution onto the network nodes, in which this section analyzes the results of simulating to evaluate the idea efficiency.

AFMD maps the tasks onto $8 \times 8$ 2D Mesh PEs and distributes the traffic based on the $X-Y$ routing algorithm, which is utilized the On/Off buffer backpressure mechanism to report releasing the input buffer. We can consider a mesh with the larger dimension that only leads to an increase in the rows of the defined traffic traces and has not affected deteriorating delay and energy consumption due to the rise in the number of the mapped tasks. The network size depends on the total number of tasks, which are mapped onto the nodes based on the pattern of the relationship between astrocyte, neuron, and dendrite. To simulate a mesh network, this work uses a cycle-accurate SystemC-based simulator and describes the relationship between the network nodes as the traffic tables and importing them to the simulator $[19,20,39]$. To deploy the simulator with a multicasting traffic feature, the researchers inspired the Noxim tool [40,41]. The simulation results demonstrate the total runtime and energy of mapping the tasks onto the network PEs and distributing their traffic onto mesh nodes using the simulator.

To analyze the efficiency of the proposed defective device detection methods, we describe the task graph (as the IoMT application) with a large number of nodes by codesign Java in MAC and Ubuntu LTS 16.04, and simulate them using the CupCarbon tool [38]. Additionally, we inject the faults onto the simulated nodes with different rates to analyze their availability and dependability to define a threshold's value for detecting a defective device by the CupCarbon. After injecting faults and estimating the availability and dependability, we re-map the tasks onto mesh processing elements due to the defective nodes and simulate by the Cycle-accurate simulator.

We first demonstrate the relationship between the activity of astrocyte and neuron cells to distribute the cholesterol and protein detection to the neurons before and after patients with Alzheimer's disease for proving the highlighted role of astrocyte operations, as shown in Figure 6a,b. The study collects a dataset of the relationship between the cells by monitoring and investigating the reported laboratory experiments of the astrocyte and neuron cells' situations based on cyclin-dependent kinase 2 (CDK2) [37]. The experimental results prove the direct relationship between the cells by computing the correlation coefficient between them that is approximately 0.9 .

To analyze the efficiency of AFMD, we inject the faults with different rates in the time slots and estimate the availability and dependability of the network's nodes by the CupCarbon tool. The simulation results prove the inverse relationship between fault rate and availability that Figure 7a-d illustrate the issue for the mesh's PEs with IndexNode number, including 11,14,35, and 38, respectively. According to the node's cost, AFMD detects the defective processing elements and tackles them based on assumption 1 and assumption 2, in which the study defines dependability or availability as the cost of the nodes.

After determining the defective nodes based on the worst hypothesis, we describe the traffic patterns based on the astrocyte operations and their relationship with the neurons to map the tasks (Vaccine distribution, vaccination, and emergency centers, and clients) onto the dependable nodes of an $8 \times 8$ 2D Mesh network. The case study describes the worst hypothesis when facing the maximum number of defective nodes, in which the communication between the mapped tasks has not failed. We raise two assumptions in facing the defective devices that are included the turn-off of the defective nodes and their interconnections (Assumption 1) and pruning them based on phagocytosis operations (Assumption 2). To evaluate the performance of the astrocyte-flow mapping and 
phagocytosis-detecting defective device, this work reports the total runtime and energy, which the simulation results prove reducing them by approximately $60.85 \%$ and $52.38 \%$ for AFM compared with the mapping the task without employing AFM pattern, as shown in Figure 8. This improvement is caused by distributing the tasks' traffic onto the network nodes in four directions and multicasting them between the PEs into the different clusters, simultaneously. According to the lack of employing the human brain neural structure in the other related works, we define a standard traffic pattern to distribute the traffic of the IoMT application based on the other studies (Without AFMD) and compare their efficiency with AFMD. Additionally, Figure 8a,b show improving communication delay and energy efficiency by approximately $6.15 \%$ and $10.26 \%$ for assumption 2 compared with assumption 1 after detecting defective nodes. After detecting the defective nodes and simulating them by the cycle-accurate tool, the relationship between processing elements demonstrates reducing the total hopcouns for assumption 2 compared with assumption 1 , which leads to performance improvement $[19,20,39]$.

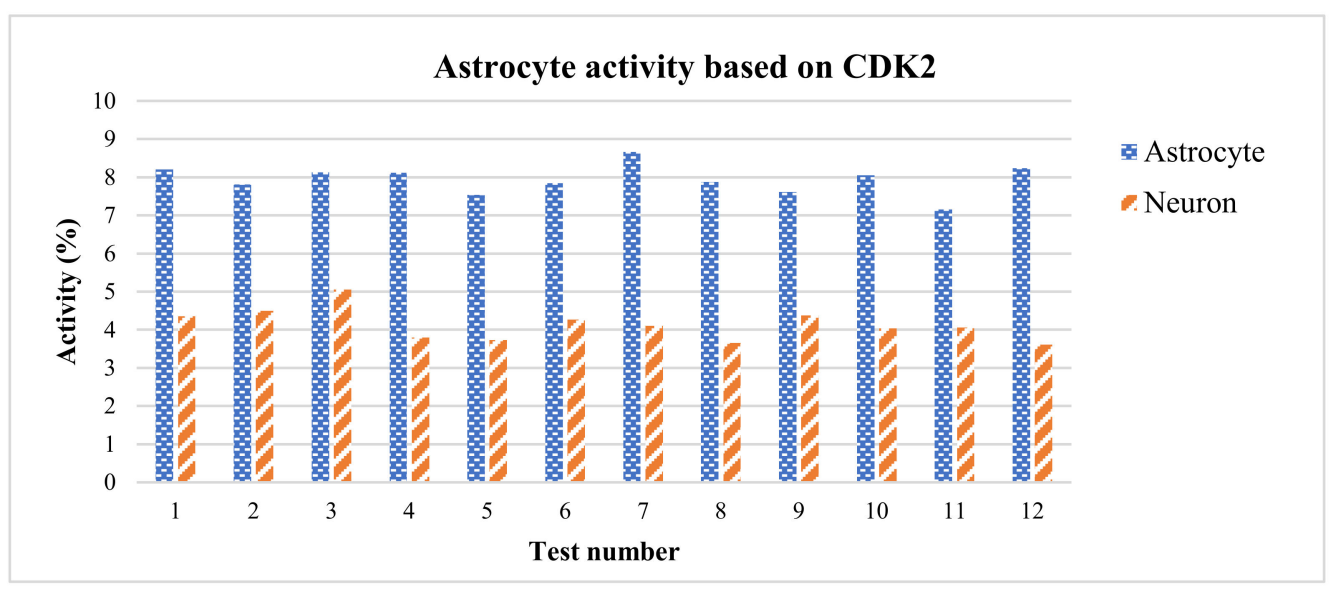

(a)

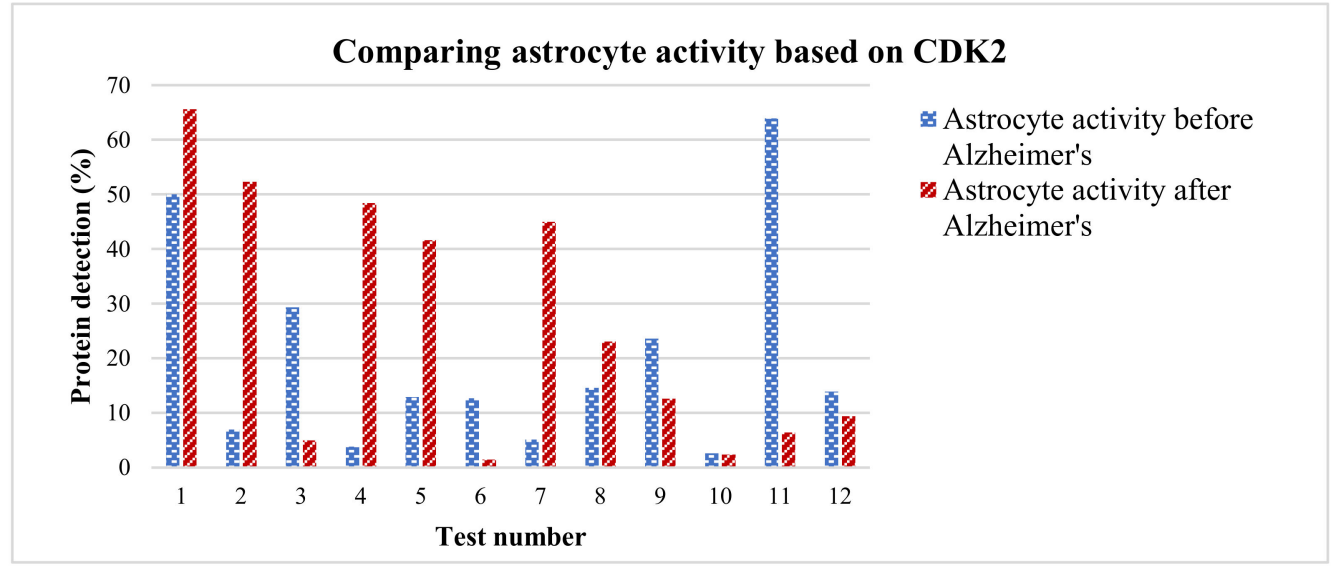

(b)

Figure 6. Analyzing the astrocyte and neuron cells' performance; (a) The relationship between the percent of astrocytes and neuron activities; (b) The detected protein before and after patients with Alzheimer's disease. 


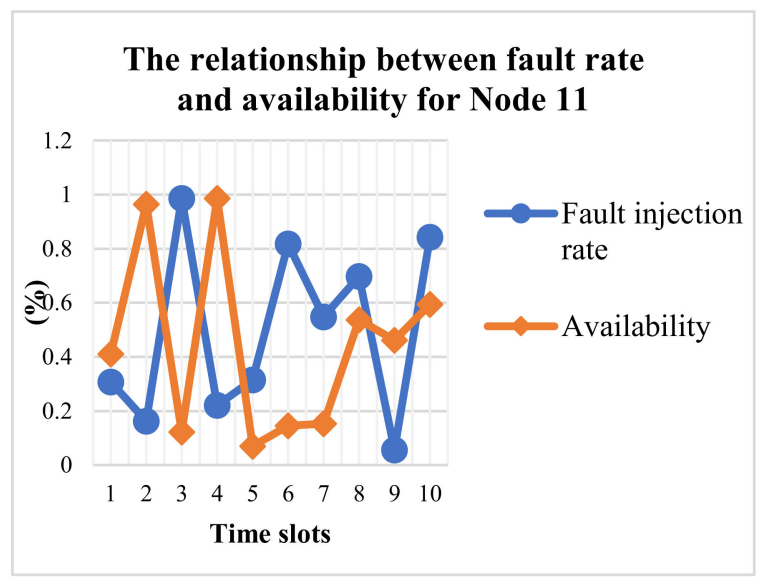

(a)

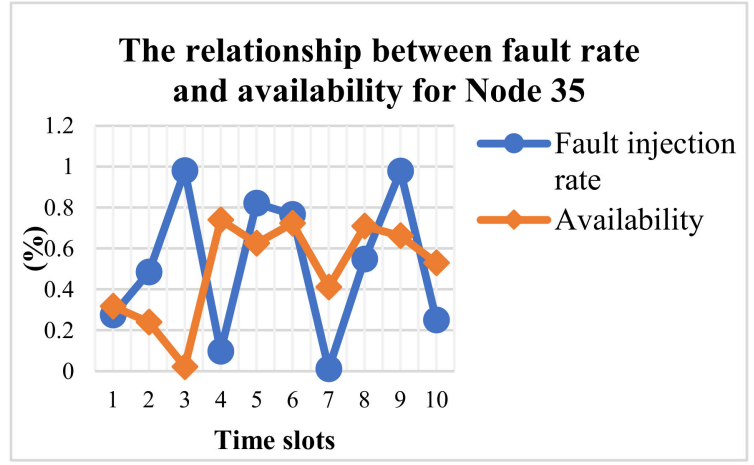

(c)

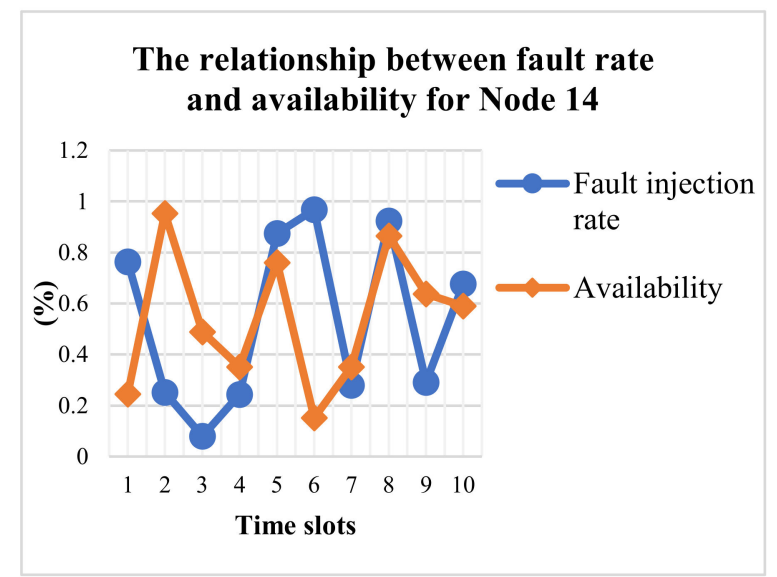

(b)

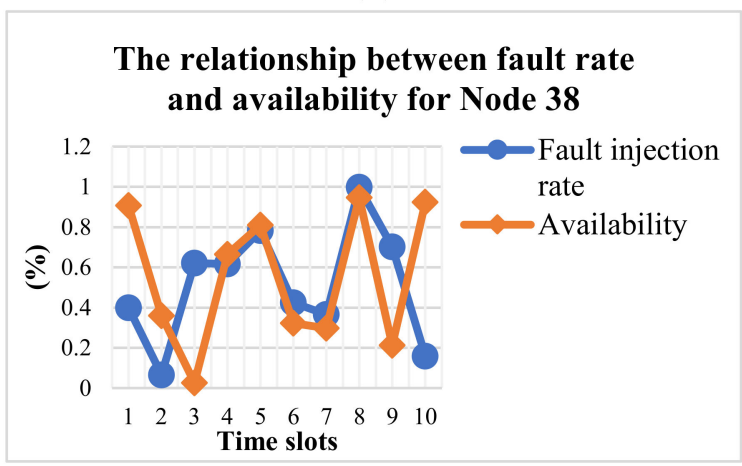

(d)

Figure 7. The relationship between fault injection rate and availability of mesh network nodes; (a) the relationship between fault injection rate and availability for node 11; (b) the relationship between fault injection rate and availability for node 14; (c) the relationship between fault injection rate and availability for node 38; (d) the relationship between fault injection rate and availability for node 35 .

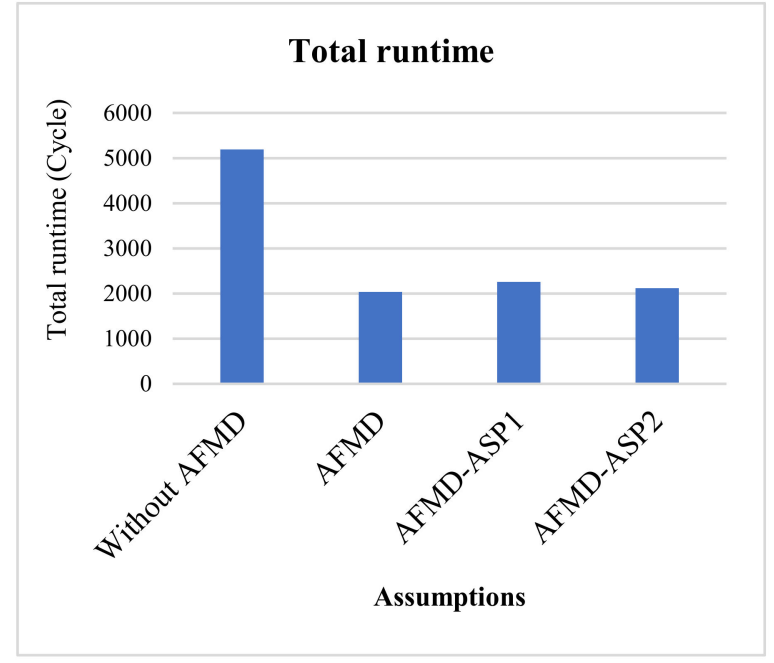

(a)

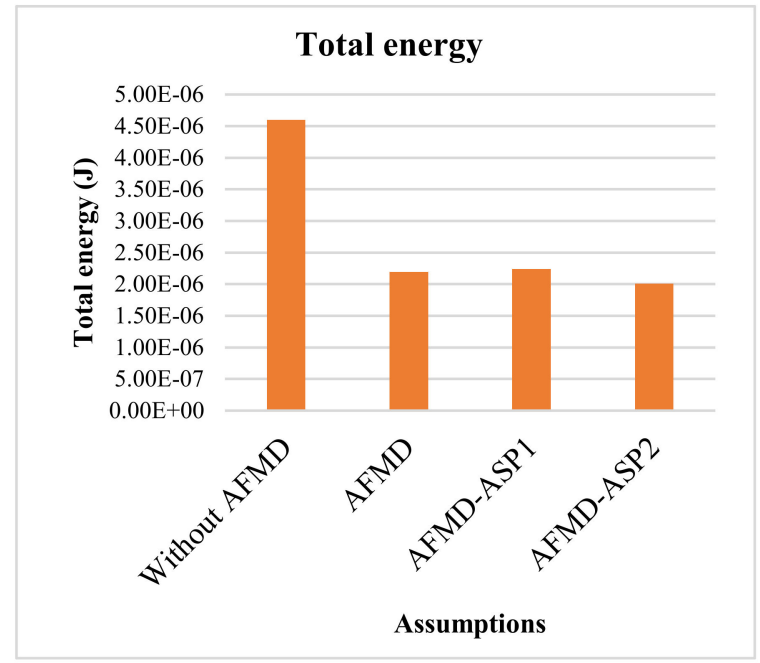

(b)

Figure 8. Comparing total runtime and energy for different situations and assumptions, including mapping the task without employing AFMD (Without AFMD), after implementing AFMD (AFMD), AFMD after detecting defective nodes based on assumption 1 (AFMD-ASP1), AFMD after detecting defective nodes based on assumption 2 (AFMD-ASP2); (a) comparing total runtime for different situations and assumptions; (b) comparing total energy for different situations and assumptions. 
This section analyzed the astrocyte operations and their relationship to neuron cells and proved the dependency between cholesterol distribution and the activity of neurons. The experimental results demonstrated reducing the activity of neuron cells after patients with Alzheimer's disease because of destructing some of them. The observations illustrated that astrocytes have an impressive effect on fairness distribute cholesterol to the neurons and support the memory's health, and operate based on a special pattern. AFMD improved the IoMT application's efficiency, which is affected by increasing the efficiency of different applications due to employing mesh-based communication infrastructures and their features. According to utilize the clustering method and phagocytosis operation, this work timely detected the defective nodes before failure the communications between a set of nodes, including successfully passing the processes of the task graph. To verify the reported observations, simulation, and experimental results, we share the collected dataset, the logs of simulators as the VCD files, and traffic patterns on Github (https:/ / github.com/yasamanhosseini/Astrocyte-flow-mapping-project accessed on 8 October 2021) [35].

\section{Discussion}

By presenting the observations and simulation results, the discussion addresses the relationship between the mentioned experimental and the proposed idea efficiency in facing IoMT challenges.

The related studies almost followed the methods to predict the different attacks to present a secure healthcare service to a requester by satisfying the patients' confidentiality records. The authentication protocols aimed to verify the doctors' legitimate and identify the shared malicious information, whereas they accessed the patients' records. The researchers proved the highlighted role of inspiring the human brain neural structures to tackle the problems of increasing the computations' complexity and neuromorphic computing-based approaches. Additionally, the studies illustrated that timely fault detection had an impressive effect on improving the quality of service in the Internet of Things and Medical Things. Since we follow the purpose of timely detecting faults and tackling them by inspiring from the human brain neural structure and analyzing its efficiency in improving IoMT-based application. This work aims to evaluate the proposed algorithm and method for timely detecting faults onto IoMT nodes by defining a task graph (Based on the Internet of Medical Things applications) and mapping it onto the mesh network due to the relationship between astrocyte and neuron cells.

In inspiring the relationship between astrocyte and neuron cells, we first analyze dependency between astrocyte cholesterol distribution and neuron activity, which results prove a direct relationship between their activities. Additionally, the observations illustrate reducing the astrocyte cells activity after infecting Alzheimer's disease caused by losing the neurons and replacing them with the amyloid plucks. The mentioned observations prove the highlighted role of balancing cholesterol distribution between neurons and nourishing them to support their health by astrocytes. Therefore, we define a task graph based on the IoMT application to map it onto the network's PEs and analyze AFM's efficiency in improving performance, which is injected the random faults onto the mesh nodes before estimating communication delay and energy consumption. After injecting faults with various rates by the CupCarbon tool, the study evaluates the dependency between the node availability and fault rate that simulation results indicate the indirect relationship between them for the different nodes of the mesh network. To assess the total runtime and energy consumption of transferring data between the network nodes, we define two assumptions of AFM-ASP1 and AFM-ASP2 for tackling the defective devices (Defective mesh nodes), which assumption 2 describes facing the faulty processing elements based on phagocytosis operations. In removing the faulty nodes and employing direct connection (Circuit switching) instead of their routers, total runtime and energy consumption are reduced for AFM-ASP2 compared with AFM-ASP1 by running the proposed algorithm. 
Other works employed the random traffic patterns to data transfer between the network nodes because of lacking the neural structure to implement the relationship between IoMT-based application nodes. We compared latency and energy efficiency for transferring data between the mesh network's PEs before and after performing AFMD. By balancing flow onto the processing elements, simulation results indicate improving the performance for astrocyte-flow mapping compared with other approaches (mapping without AFMD).

\section{Conclusions}

The Internet of Medical Things-infrastructures supports a huge number of sensors, patients, different healthcare service providers that are led to increase the complexity of the relationship between them and their computational operations. The issue is challenging for IoMT to balance the flow between its nodes in the different layers and detect a defective device before failure the communications and losing trust in the relationship between service providers and requesters. Some case studies addressed the human brain's structure-based methods to face the problems caused by increasing the complexity in different applications such as machine learning algorithms, decision-making in IoT, and simulating the body language. According to the highlighted role of astrocyte cells as a part of the human brain's neuron structure, a study can utilize the cell features in distributing cholesterol into the neurons to tackle the challenge of IoMT's complexity. Since we proposed a flow mapping method to distribute an IoMT application's traffic onto mesh network processing elements and detected the defective nodes by inspiring the astrocyte and phagocytosis operations. Our work defined a task graph based on the Internet of Medical Things application (Vaccination COVID-19) and the relationship between astrocyte, neuron, and dendrite for evaluating the idea efficiency in improving IoMT performance. We improved its performance by balancing flow between its nodes and detecting a defective device before failure the communication between a set of nodes and losing trust between them. The work achieved the result by estimating communication delay and energy consumption caused by data transfer between IoMT nodes before and after performing the proposed algorithm.

By inspiring the human brain's neural structure, we will improve the Internet of Medical Things-based applications' performance in different aspects. We can predict the defective device's area of the different IoMT applications by analyzing the patterns of distributing proteins (such as $\alpha, \beta, \alpha \beta$, and bach1) and replacing neurons by them, which depends on the brain disease types (Such as Parkinson, depression, anhedonia, and Alzheimer's).

Author Contributions: Conceptualization, A.M.R., R.A.N., S.A., S.Y.H.M., M.A., M.H. and K.S.; Data curation, A.M.R., R.A.N., S.A., S.Y.H.M., M.A., M.H. and K.S.; Formal analysis, A.M.R., R.A.N., S.A., S.Y.H.M., M.A., M.H. and K.S.; Funding acquisition, A.M.R., R.A.N., S.A., S.Y.H.M., M.A., M.H. and K.S.; Investigation, A.M.R., R.A.N., S.A., S.Y.H.M., M.A., M.H. and K.S.; Methodology, A.M.R., R.A.N., S.A., S.Y.H.M., M.A., M.H. and K.S.; Project administration, A.M.R., R.A.N., S.A., S.Y.H.M., M.A., M.H. and K.S.; Resources, A.M.R., R.A.N., S.A., S.Y.H.M., M.A., M.H. and K.S.; Software, A.M.R., R.A.N., S.A., S.Y.H.M., M.A., M.H. and K.S.; Supervision, A.M.R., R.A.N., S.A., S.Y.H.M., M.A., M.H. and K.S.; Validation, A.M.R., R.A.N., S.A., S.Y.H.M., M.A., M.H. and K.S.; Visualization, A.M.R., R.A.N., S.A., S.Y.H.M., M.A., M.H. and K.S.; Writing-original draft, A.M.R., R.A.N., S.A., S.Y.H.M., M.A., M.H. and K.S.; Writing-review \& editing, A.M.R., R.A.N., S.A., S.Y.H.M., M.A., M.H. and K.S. All authors have read and agreed to the published version of the manuscript.

Funding: The authors acknowledge that this research was supported by Xiamen University Malaysia Research Fund (Grant No: XMUMRF/2019-C4/IECE/0012).

Informed Consent Statement: We did not utilize the patients for the research.

Conflicts of Interest: We declare that this manuscript is original, has not been published before and is not currently being considered for publication elsewhere. 


\section{References}

1. Nguyen, T.A.; Min, D.; Choi, E.; Lee, J.W. Dependability and Security Quantification of an Internet of Medical Things Infrastructure based on Cloud-Fog-Edge Continuum for Healthcare Monitoring using Hierarchical Models. IEEE Internet Things J. 2021, 8 , 15704-15748. [CrossRef]

2. Kumar, A.; Sharma, K.; Sharma, A. Genetically optimized Fuzzy C-means data clustering of IoMT-based biomarkers for fast affective state recognition in intelligent edge analytics. Appl. Soft Comput. 2021, 109, 107525. [CrossRef]

3. Yadav, V.K.; Yadav, R.K.; Verma, S.; Venkatesan, S. CP2EH: A comprehensive privacy-preserving e-health scheme over cloud. J. Supercomput. 2021, 1-31. [CrossRef]

4. Almiani, M.; AbuGhazleh, A.; Al-Rahayfeh, A.; Atiewi, S.; Razaque, A. Deep recurrent neural network for IoT intrusion detection system. Simul. Model. Pr. Theory 2020, 101, 102031. [CrossRef]

5. Pustokhina, I.V.; Pustokhin, D.A.; Gupta, D.; Khanna, A.; Shankar, K.; Nguyen, G.N. An effective training scheme for deep neural network in edge computing enabled Internet of medical things (IoMT) systems. IEEE Access 2020, 8, 107112-107123. [CrossRef]

6. Davies, M.; Wild, A.; Orchard, G.; Sandamirskaya, Y.; Guerra, G.A.F.; Joshi, P.; Plank, P.; Risbud, S.R. Advancing Neuromorphic Computing with Loihi: A Survey of Results and Outlook. Proc. IEEE 2021, 109, 911-934. [CrossRef]

7. Kwon, H.; Samajdar, A.; Krishna, T. Maeri: Enabling flexible dataflow mapping over dnn accelerators via reconfigurable interconnects. ACM SIGPLAN Not. 2018, 53, 461-475. [CrossRef]

8. Kwon, H.; Pellauer, M.; Parashar, A.; Krishna, T. Flexion: A Quantitative Metric for Flexibility in DNN Accelerators. IEEE Comput. Arch. Lett. 2021, 20, 1-4. [CrossRef]

9. Kwon, H.; Chatarasi, P.; Sarkar, V.; Krishna, T.; Pellauer, M.; Parashar, A. MAESTRO: A Data-Centric Approach to Understand Reuse, Performance, and Hardware Cost of DNN Mappings. IEEE Micro 2020, 40, 20-29. [CrossRef]

10. Piccialli, F.; Casolla, G.; Cuomo, S.; Giampaolo, F.; Di Cola, V.S. Decision Making in IoT Environment through Unsupervised Learning. IEEE Intell. Syst. 2020, 35, 27-35. [CrossRef]

11. Gope, P.; Gheraibia, Y.; Kabir, S.; Sikdar, B. A secure IoT-based modern healthcare system with fault-tolerant decision making process. IEEE J. Biomed. Health Inform. 2020, 25, 862-873. [CrossRef]

12. Dilibal, Ç. Development of edge-IoMT computing architecture for smart healthcare monitoring platform. In Proceedings of the 2020 4th International Symposium on Multidisciplinary Studies and Innovative Technologies (ISMSIT), Istanbul, Turkey, 22-24 October 2020; pp. 1-4.

13. Guan, Y.; Ohsawa, T. Co-Design of Binary Processing in Memory ReRAM Array and DNN Model Optimization Algorithm. IEICE Trans. Electron. 2020, 103, 685-692. [CrossRef]

14. Rahmani, A.M.; Mirmahaleh, S.Y. A predictor circuit and a delay-aware algorithm for identifying data transfer pattern on NoC-based communication networks. Microelectron. J. 2021, 116, 105250. [CrossRef]

15. Zhu, Z.; Sun, H.; Qiu, K.; Xia, L.; Krishnan, G.; Dai, G.; Niu, D.; Chen, X.; Hu, X.S.; Cao, Y.; et al. MNSIM 2.0: A Behavior-Level Modeling Tool for Memristor-based Neuromorphic Computing Systems. In MNSIM 2.0: A Behavior-Level Modeling Tool for Memristor-based Neuromorphic Computing Systems. In Proceedings of the 2020 on Great Lakes Symposium on VLSI, online, 7 September 2020; ACM Press: New York, NY, USA; pp. 83-88.

16. Ceolini, E.; Frenkel, C.; Shrestha, S.B.; Taverni, G.; Khacef, L.; Payvand, M.; Donati, E. Hand-Gesture Recognition Based on EMG and Event-Based Camera Sensor Fusion: A Benchmark in Neuromorphic Computing. Front. Neurosci. 2020, 14, 637. [CrossRef]

17. Montesinos, J.; Pera, M.; Larrea, D.; Guardia-Laguarta, C.; Agrawal, R.R.; Velasco, K.R.; Yun, T.D.; Stavrovskaya, I.G.; Xu, Y.; Koo, S.Y.; et al. The Alzheimer's disease-associated C99 fragment of APP regulates cellular cholesterol trafficking. EMBO J. 2020, 39, e103791. [CrossRef] [PubMed]

18. Desale, S.E.; Chinnathambi, S. Role of dietary fatty acids in microglial polarization in Alzheimer's disease. J. Neuroinflammation 2020, 17, 1-14. [CrossRef] [PubMed]

19. Mirmahaleh, S.Y.H.; Reshadi, M.; Shabani, H.; Guo, X.; Bagherzadeh, N. Flow mapping and data distribution on mesh-based deep learning accelerator. In Proceedings of the 13th IEEE/ACM International Symposium on Networks-on-Chip, New York, NY, USA, 17 October 2019; p. 13.

20. Mirmahaleh, S.Y.H.; Reshadi, M.; Bagherzadeh, N. Flow mapping on mesh-based deep learning accelerator. J. Parallel Distrib. Comput. 2020, 144, 80-97. [CrossRef]

21. Mesbahi, M.R.; Rahmani, A.M.; Hosseinzadeh, M. Highly reliable architecture using the $80 / 20$ rule in cloud computing datacenters. Future Gener. Comput. Syst. 2017, 77, 77-86. [CrossRef]

22. Javaheri, D.; Hosseinzadeh, M.; Rahmani, A.M. Detection and elimination of spyware and ransomware by intercepting kernellevel system routines. IEEE Access 2018, 6, 78321-78332. [CrossRef]

23. Heikalabad, S.R.; Navin, A.H.; Hosseinzadeh, M.; Oladghaffari, T. Midpoint memory: A special memory structure for dataoriented models implementation. J. Circ. Syst. Comput. 2015, 24, 1550063. [CrossRef]

24. Kumar, P.; Gupta, G.P.; Tripathi, R. An ensemble learning and fog-cloud architecture-driven cyber-attack detection framework for IoMT networks. Comput. Commun. 2021, 166, 110-124. [CrossRef]

25. Rahman, M.; Jahankhani, H. Security vulnerabilities in existing security mechanisms for iomt and potential solutions for mitigating cyber-attacks. In Information Security Technologies for Controlling Pandemics 2021; Springer: Cham, Switzerland, 2021; pp. 307-334. 
26. Khan, S.; Akhunzada, A. A hybrid DL-driven intelligent SDN-enabled malware detection framework for Internet of Medical Things (IoMT). Comput. Commun. 2021, 170, 209-216. [CrossRef]

27. Chaabouni, N.; Mosbah, M.; Zemmari, A.; Sauvignac, C.; Faruki, P. Network Intrusion Detection for IoT Security Based on Learning Techniques. IEEE Commun. Surv. Tutorials 2019, 21, 2671-2701. [CrossRef]

28. Xiong, H.; Jin, C.; Alazab, M.; Yeh, K.-H.; Wang, H.; Gadekallu, T.R.R.; Wang, W.; Su, C. On the Design of Blockchain-based ECDSA with Fault-tolerant Batch Verication Protocol for Blockchain-enabled IoMT. IEEE J. Biomed. Health Inform. 2021, 1. [CrossRef]

29. Das, A.K.; Bera, B.; Giri, D. AI and Blockchain-Based Cloud-Assisted Secure Vaccine Distribution and Tracking in IoMT-Enabled COVID-19 Environment. IEEE Internet Things Mag. 2021, 4, 26-32. [CrossRef]

30. Kumar, S.; Ahmadian, A.; Kumar, R.; Kumar, D.; Singh, J.; Baleanu, D.; Salimi, M. An Efficient Numerical Method for Fractional SIR Epidemic Model of Infectious Disease by Using Bernstein Wavelets. Mathematics 2020, 8, 558. [CrossRef]

31. Wang, P.; Wang, J.; Wei, G.; Wei, C. Similarity measures of q-rung orthopair fuzzy sets based on cosine function and their applications. Mathematics 2019, 340, 340. [CrossRef]

32. Khan, S.R.; Sikandar, M.; Almogren, A.; Din, I.U.; Guerrieri, A.; Fortino, G. IoMT-based computational approach for detecting brain tumor. Future Gener. Comput. Syst. 2020, 109, 360-367. [CrossRef]

33. Deng, W.; Shi, Q.; Wang, M.; Zheng, B.; Ning, N. Deep Learning-Based HCNN and CRF-RRNN Model for Brain Tumor Segmentation. IEEE Access 2020, 8, 26665-26675. [CrossRef]

34. Liu, J.; Harkin, J.; Maguire, L.; McDaid, L.J.; Wade, J.J.; Martin, G. Scalable Networks-on-Chip Interconnected Architecture for Astrocyte-Neuron Networks. IEEE Trans. Circuits Syst. I Regul. Pap. 2016, 63, 2290-2303. [CrossRef]

35. Dataset. Available online: https://github.com/yasamanhosseini/Astrocyte-flow-mapping-project (accessed on 8 October 2021).

36. Coronavirus (COVID-19) Vaccinations. Available online: https://ourworldindata.org/covid-vaccinations (accessed on 8 October 2021).

37. BioGPS. Available online: http://biogps.org/dataset/tag/astrocyte/ (accessed on 8 October 2021).

38. Simulator. Available online: http:// cupcarbon.com/ (accessed on 8 October 2021).

39. Mirmahaleh, S.Y.H.; Reshadi, M.; Bagherzadeh, N.; Khademzadeh, A. Data scheduling and placement in deep learning accelerator. Clust. Comput. 2021, 24, 3651-3669. [CrossRef]

40. Simulator. Available online: https://github.com/davidepatti/noxim (accessed on 8 October 2021).

41. Catania, V.; Mineo, A.; Monteleone, S.; Palesi, M.; Patti, D. Cycle-Accurate Network on Chip Simulation with Noxim. ACM Trans. Model. Comput. Simul. 2016, 27, 1-25. [CrossRef] 